\title{
Twelve months clinical outcome after bioresorbable vascular scaffold implantation in patients with stable angina and acute coronary syndrome. Data from the Polish National Registry
}

\author{
Łukasz Rzeszutko' ${ }^{1}$, Zbigniew Siudak², Tomasz Tokarek², Krzysztof Plens ${ }^{3}$, Adrian Włodarczak', \\ Andrzej Lekston ${ }^{5}$, Andrzej Ochała ${ }^{6}$, Robert J. Gil ${ }^{7,8}$, Wojciech Balak ${ }^{9}$, Dariusz Dudek ${ }^{2}$ \\ ${ }^{1} 2^{\text {nd }}$ Department of Cardiology, Institute of Cardiology, Jagiellonian University, Medical College, Krakow, Poland \\ ${ }^{2}$ Department of Interventional Cardiology, Institute of Cardiology, Jagiellonian University Medical College, Krakow, Poland \\ ${ }^{3}$ Krakow Cardiovascular Research Institute, Krakow, Poland \\ ${ }^{4}$ Miedziowe Centrum Zdrowia S.A., Lublin, Poland \\ ${ }^{5} 3^{\text {rd }}$ Department and Clinical Unit of Cardiology, Silesian Centre for Heart Diseases, Medical University of Silesia, Zabrze, Poland \\ ${ }^{6} 3^{\text {rd }}$ Department of Cardiology, Medical University of Silesia, Katowice, Poland \\ ${ }^{7}$ Invasive Cardiology Clinic, Central Clinical Hospital of the Internal Affairs and Administration Ministry, Warsaw, Poland \\ ${ }^{8}$ Institute of Experimental and Clinical Medicine, Polish Academy of Science, Warsaw, Poland \\ ${ }^{9} 2^{\text {nd }}$ Department of Cardiology, Ludwik Rydygier Collegium Medicum in Bydgoszcz, Nicolaus Copernicus University in Torun, Poland
}

Adv Interv Cardiol 2016; 12, 2 (44): 108-115

DOI: 10.5114 /aic.2016.59360

\begin{abstract}
A bstract
Introduction: There are limited data describing bioresorbable vascular scaffold (BVS) implantation in complex lesions. Only short-term clinical outcomes are available for patients with acute coronary syndrome (ACS).

Aim: To evaluate 12-month clinical outcome, safety and effectiveness of BVS implantation in complex lesions and in stable angina (SA) or ACS.

Material and methods: Five hundred ninety-one patients with SA/ACS were enrolled between October 2012 and November 2013 in 30 invasive cardiology centres in Poland. At least one BVS implantation during percutaneous coronary intervention (PCI) was the only inclusion criteria. The clinical endpoint was the occurrence of a major adverse cardiovascular event (MACE) (all-cause death, myocardial infarction (MI), clinically driven target lesion revascularisation (TLR) with urgent PCI or target vessel revascularisation (TVR) with urgent coronary artery bypass grafting (CABG)) and device-oriented composite endpoint (DOCE) (cardiac death, urgent target vessel revascularisation with PCI/CABG, target vessel MI) during 12-month follow-up.

Results: After 12 months TLR with urgent PCI was significantly more often reported in patients with diagnosed UA (4.59\%; $p<0.02)$ in comparison with other $\mathrm{PCl}$ indications. No significant differences were found in terms of composite MACE endpoint, cumulative MACE $(p=0.09)$, stent thrombosis $(p=0.2)$ or restenosis $(p=0.2)$. There were no significant differences in cumulative MACE and composite MACE endpoint between patients with no/mild versus moderate/severe tortuosity and no/mild versus moderate/severe calcification of the target vessel. No significant difference was found between groups of patients with or without bifurcation of the target vessel. Device-oriented composite endpoint was significantly more often reported in the ACS group (3.2\% vs. $0.47 \%$; $p$ 0.03), most frequently in patients with diagnosed UA $(5.5 \%)$.

Conclusions: Bioresorbable vascular scaffold can be successfully and safely used for ACS treatment and in lesions of higher complexity.
\end{abstract}

Key words: stent, calcified, bifurcation, tortuosity, complex lesion.

\section{Introduction}

The introduction of the bioresorbable vascular scaffold (BVS) is a new approach in interventional cardiology
[1]. It provides transient vessel support and drug delivery without the potential long-term limitations of metallic implants [2]. Prolonged contact with a foreign material

\section{Corresponding author:}

Prof. Dariusz Dudek MD, PhD, Department of Interventional Cardiology, Institute of Cardiology, Jagiellonian University Medical College, 17 Kopernika St, 31-501 Krakow, Poland, phone: +48 1242471 81, e-mail: mcdudek@cyfronet.pl

Received: 20.11.2015, accepted: 11.02.2016. 
can lead to limited vasomotion, chronic inflammation, late expansive remodelling and late thrombosis, and may preclude surgical revascularisation. After complete bioresorption in 2-3 years $[3,4]$, the BVS leaves the vessel covered with a healthy endothelium and restored vasomotion [5]. Data presented in the ABSORB trials showed the efficacy and safety of BVS [6] and their unique advantage in the restoration of vasomotion [7]. Long-term results are still limited to a small number of patients [7]. There are limited data describing BVS implantation in complex lesions such as bifurcations, calcified lesions or severely tortuous vessels. Only short-term clinical outcomes are available for patients with acute coronary syndrome (ACS) [8].

\section{Aim}

We sought to evaluate 12-month clinical outcome, safety and effectiveness of BVS implantation in lesions of higher complexity and in the setting of both stable angina (SA) and ACS in a Polish contemporary registry study.

\section{Material and methods}

This report represents a one-arm retrospective observational registry study, which enrolled patients in 30 invasive cardiology centres in Poland. Data describing baseline clinical and demographic characteristics of the patients, past medical history, angiography and percutaneous coronary intervention ( $\mathrm{PCl}$ ) details as well as periprocedural and in-hospital outcomes have been reported in the previous paper [9]. Patients with at least one BVS implantation during the index $\mathrm{PCI}$ were included in the registry. There were no additional inclusion or exclusion criteria. The study group consisted of 591 consecutive patients who underwent PCI between October 2012 and November 2013. The 12-month follow-up was completed in $79 \%$ (468 patients). The bioethics committee of the Jagiellonian University in Krakow approved the protocol of the registry. The clinical endpoint for the study was the occurrence of a major adverse cardiovascular event (MACE) and device-oriented composite endpoint (DOCE) during 12-month follow-up. The MACE was defined as all-cause death, myocardial infarction (MI), clinically driven target lesion revascularisation (TLR) with urgent $\mathrm{PCl}$ or target vessel revascularisation (TVR) with urgent coronary artery bypass grafting (CABG). Device-oriented composite endpoint included cardiac death, TVR with urgent $\mathrm{PCl}$ or $\mathrm{CABG}$ and target vessel MI. All individual components of events and device thrombosis were defined by the Academic Research Consortium (ARC) criteria [10]. Occurrences of adverse events were assessed at regular clinical follow-up in hospital or by standardised telephone interview.

\section{Statistical analysis}

Standard descriptive statistics were used in the analysis. Quantitative variables were described using means and standard deviation. Categorical variables were presented with counts and as percentages. The level of statistical significance was set at $p \leq 0.05$. All calculations were done with JMP 9.0.0 software (SAS Institute Inc., Cary, NC). This study was executed in cooperation with an independent contract research organisation - Krakow Cardiovascular Research Institute (KCRI, Poland).

\section{Results}

Complete baseline clinical and demographic characteristics of the included 591 patients were reported previously [9]. Technical delivery success was achieved in $100 \%$ of cases. One-year clinical outcomes of included patients are summarised in Table I. After 12 months there were no significant differences in rates of stent thrombosis (ST) $(p=0.2)$, stent restenosis (SR) $(p=0.2)$, all-cause death $(p=0.2)$, occurrence of MI $(p=0.9)$, TVR with urgent $C A B G$ or cumulative MACE $(p=0.09)$ in comparison between patients with SA, unstable angina (UA), non-ST elevation myocardial infarction (NSTEMI) and ST elevation myocardial infarction (STEMI). Target lesion revascularisation with urgent $\mathrm{PCl}$ was significantly more often performed in patients with diagnosed UA in comparison with SA, STEMI and NSTEMI groups (respectively: $4.59 \%$ vs. $0.47 \%$ vs. $1.82 \%$ vs. $0.0 \% ; p<0.02$ ). There were no significant differences in cumulative MACE and composite MACE endpoint between patients with diagnosed ACS and SA (Table I). Kaplan-Meier curves for MACE incidence are presented in Figure 1. Device-oriented composite endpoint was significantly more often reported in the ACS group (Table I), the most often in patients with UA in comparison with other $\mathrm{PCl}$ indications (respectively: $5.5 \%$ vs. $0.47 \%$ vs. $0.0 \%$ vs. $3.7 \%$; $p<0.006$ ). Kaplan-Meier curves for DOCE incidence are presented in Figure 2. No significant differences were observed in clinical outcome in comparison between patients with simple (ACC/ AHA type A or B1) and complex lesions (ACC/AHA type B2 or $C$ ). There were no significant differences in cumulative DOCE, cumulative MACE and composite MACE endpoint between patients with no or mild vs. moderate or severe tortuosity of the target vessel (Table II). Furthermore, no significant differences in 12 months clinical outcome were observed in comparison of patients with no or mild vs. moderate or severe calcification in the target vessel (Table III). Likewise, data were obtained between groups of patients with or without bifurcation of the target vessel (Table IV). The first case of ST occurred within 30 days after the procedure (STEMI group), the second at 90 days after BVS implantation (UA group). The majority of events occurred within six months after BVS implantation.

\section{Discussion}

According to the present data, DOCE was significantly more frequent in the ACS group, with the highest incidence in patients with diagnosed UA. Furthermore, TLR 
Table I. Clinical outcome, device oriented composite endpoint and major adverse cardiovascular events after 12 months observation of all included patients and in comparison of acute coronary syndrome with stable angina. Follow-up was available in 468 patients. Total MACE number is not equal to added components. Few events may occur in 1 patients

\begin{tabular}{|c|c|c|c|c|}
\hline Parameter & All patients $(n=468)$ & ACS $(n=250)$ & SA $(n=215)$ & $P$-value ${ }^{a}$ \\
\hline DOCE & $9(1.93 \%)$ & $8(3.2 \%)$ & $1(0.47 \%)$ & $<0.03$ \\
\hline MACE & $14(3.03 \%)$ & $11(4.44 \%)$ & $3(1.4 \%)$ & 0.06 \\
\hline Death & $2(0.43 \%)$ & $2(0.8 \%)$ & $0(0.0 \%)$ & 0.5 \\
\hline MI & $8(1.73 \%)$ & $5(2.01 \%)$ & $3(1.4 \%)$ & 0.7 \\
\hline TLR with urgent $\mathrm{PCl}$ & $7(1.51 \%)$ & $6(2.41 \%)$ & $1(0.47 \%)$ & 0.1 \\
\hline TVR with urgent $C A B G$ & $0(0.00 \%)$ & $0(0.00 \%)$ & $0(0.00 \%)$ & - \\
\hline Stent thrombosis & $2(0.43 \%)$ & $2(0.8 \%)$ & $0(0.0 \%)$ & 0.5 \\
\hline Stent restenosis & $10(2.16 \%)$ & 8 (3.21\%) & $2(0.93 \%)$ & 0.1 \\
\hline
\end{tabular}

P-value ${ }^{a}$-statistical significance for comparison of ACS vs. no ACS. ACS - acute coronary syndrome, CABG - coronary artery bypass grafting, DOCE - device-oriented composite endpoint, MACE - major adverse cardiovascular event, MI-myocardial infarction, $P C I$ - percutaneous coronary interventions, SA - stable angina, TLR target lesion revascularisation, TVR - target vessel revascularisation.

with urgent $\mathrm{PCl}$ was significantly more frequent in the UA group. A low rate of events was observed in the observation period. Furthermore, BVS implantation to more complex lesions seems to be safe and have no negative impact after 12 months of observation. Several studies have reported a MACE rate between $2.6 \%$ and $10.7 \%$ [11]. Two recently published large analyses presented MACE in $5.0 \%$ after 12 months of observation [12, 13]. Our study showed a lower incidence of MACE in comparison with the above-mentioned studies. The composite MACE endpoint rate also compares favourably with most of the available BVS implantation data. All-cause mortality after 6 months of observation was reported in $1.3 \%$ in a recent all-comers registry [14]. Data from two other studies showed an incidence of all-cause death of $0.8 \%$ after 12 months of follow-up $[15,16]$. A lower incidence of all-cause mortality was observed in our study in comparison with those studies; however, in the POLAR ACS and TROFI II trial no deaths occurred during one year and 6 months of follow-up, respectively $[6,17]$. Data from a meta-analysis of BVS use showed $\mathrm{MI}$ in $5.2 \%$ and TLR in 3.0\% [15]. The GHOST-EU registry also demonstrated higher incidence of these events in comparison with our study [14]. Device-oriented events are also crucial for evaluation of scaffold implantation efficacy and safety. Device-oriented composite endpoint was reported in $4.7 \%$ and $4.1 \%$ of patients from two recently published analyses [12,18]. Superior results were obtained in our study in terms of DOCE in comparison with large studies; however, another study showed occurrence in 1.1\% [17]. ST appeared to be the most important limitation of polymeric scaffolds in the early phase after implantation [11]. The GHOST-EU registry reported definite/probable ST after 30 days and 12 months in $1.5 \%$ and $3.4 \%$, re- spectively [14]. A recent meta-analysis demonstrated incidence in $1.26 \%$ of included patients [15]. In both studies the most reported events occurred during first 30 days after the procedure, as in our research [14, 15]. Another study reported definite/probable ST in around $1.1 \%$ [12]. ABSORB EXTEND showed similar incidence after 3 years of observation [19]. Recent research with 290 consecutive STEMI patients demonstrated definite/probable ST in $2.1 \%$ and $2.4 \%$ after 30 days and 12 months of observation, respectively [18]. Despite enrolment of patients with more complex lesions, data in our study compare favourably with above-mentioned studies. The optimal clinical results from the current study could be the result of the aggressive strategy of lesion preparation and post-dilatation during scaffold delivery [9]. Direct comparisons between our analysis and different patient populations from other studies cannot be made; however, the low rates of ST, MACE, composite MACE endpoint and DOCE during 12 months of observation in our cohort supports this strategy of BVS implantation. Furthermore, two other studies using the offensive strategy of lesion preparation and routine high-pressure postdilation with intracoronary imaging showed no ST events despite inclusion of complex lesions [20-22]. Adequate antiplatelet treatment is also important to achieve an optimal clinical outcome. For patients treated with BVS more aggressive antiplatelet therapy was postulated [11]. In a recent study clopidogrel, prasugrel and ticagrelor were prescribed in $73.2 \%, 26.2 \%$ and $0.6 \%$ of patients, respectively [14]. Compared to our previous paper, ticagrelor was prescribed at discharge more often in the current study (6\%) [9]. However, the TROFI II trial demonstrated optimal clinical outcomes after 6 months of observation with ticagrelor use at discharge in $46.2 \%$ and prasugrel in 
A

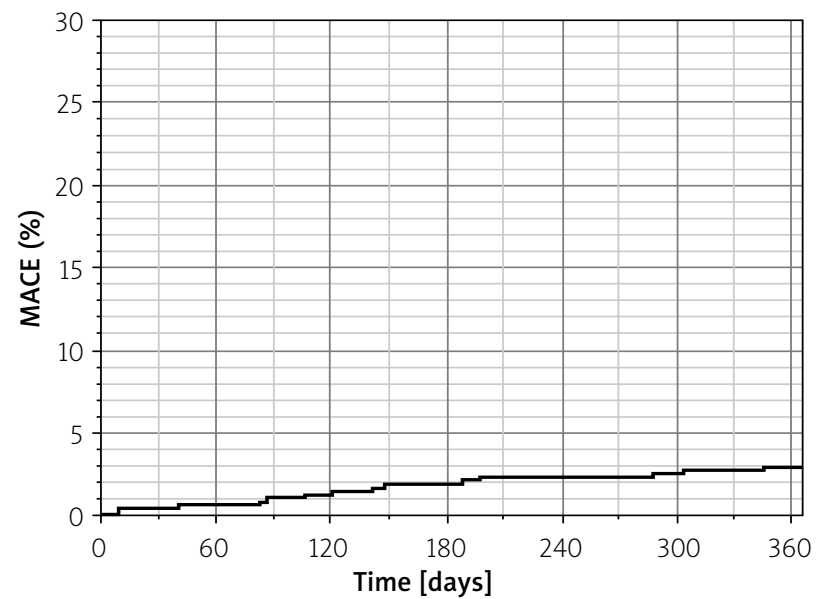

C

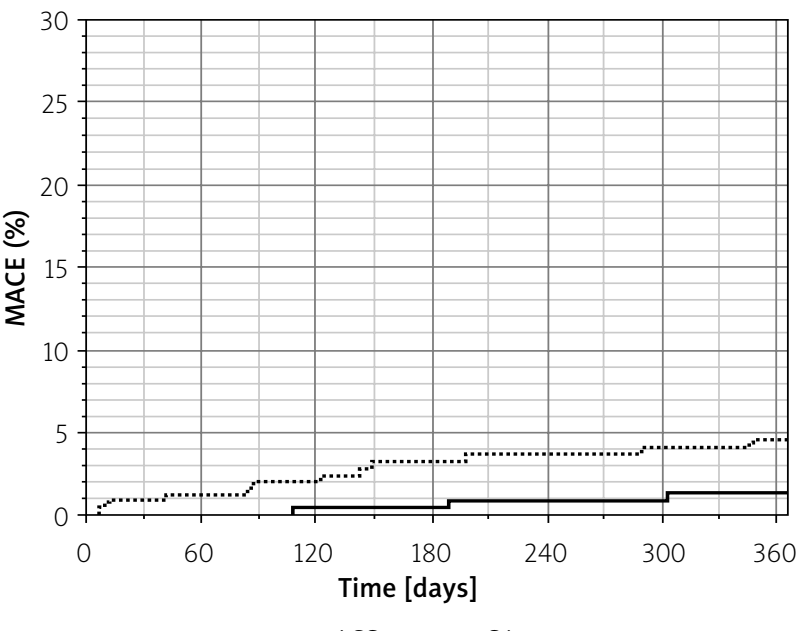

$\mathrm{E}$

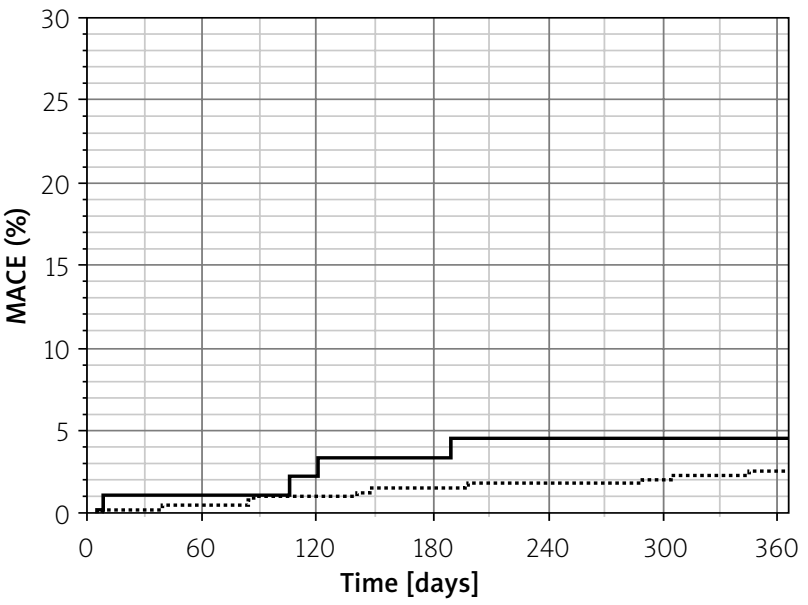

....... No calcification and mild _ Moderate and heavy
B

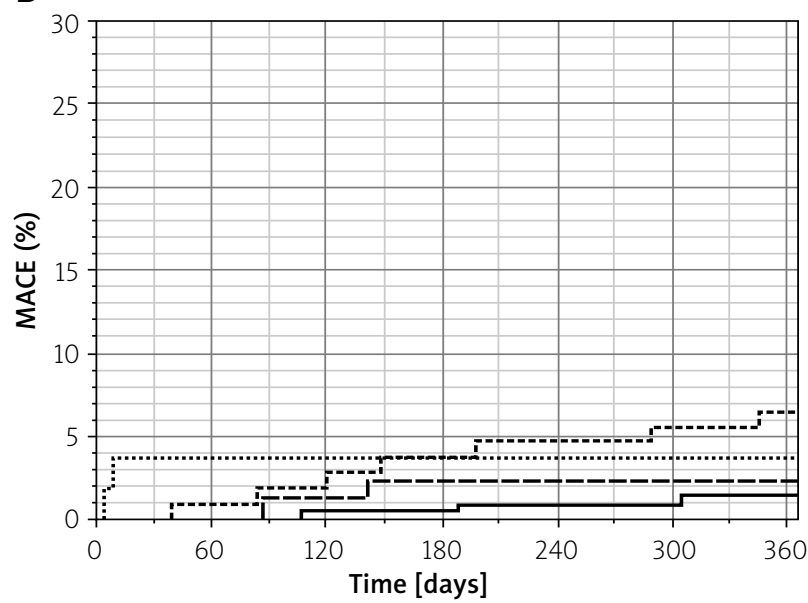

...... STEMI - - NSTEMI

_ Stable angina _.--.. Unstable angina

D

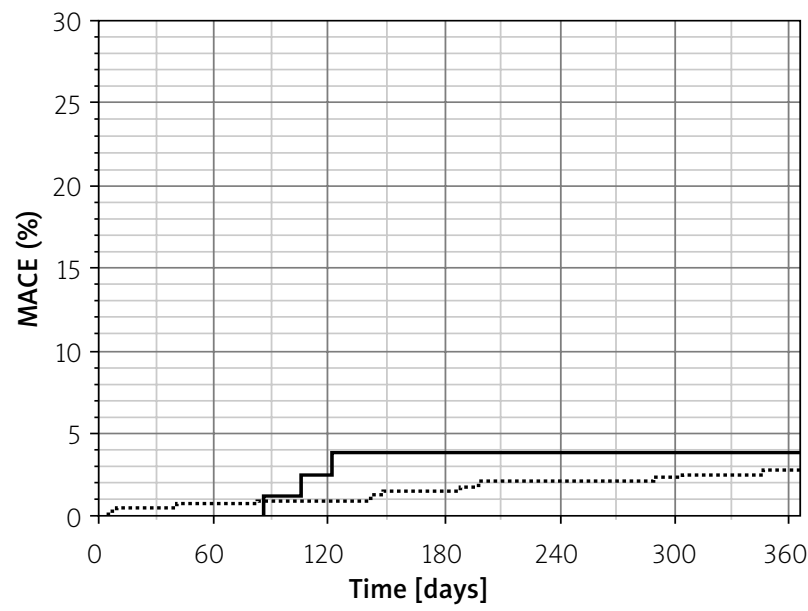

...... Not tortuous and mild _ Moderate and severe

$\mathbf{F}$

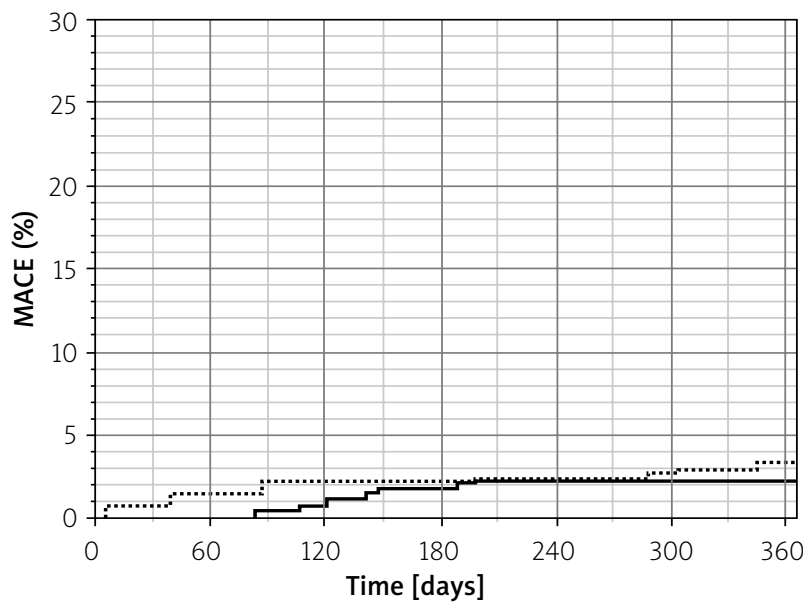

....... Yes No

Figure 1. Kaplan-Meier curves for major adverse cardiovascular event incidence: $A$ - MACE in all included patients, $\mathbf{B}-$ MACE in comparison of PCl indications $(p=0.09), \mathbf{C}-$ MACE in comparison of ACS vs. SA $(p=0.06)$, D - MACE in comparison between patients with no or mild versus moderate or severe tortuosity at target vessel $(p=0.6), E-M A C E$ in comparison between patients with no or mild vs. moderate or heavy calcification at target vessel $(p=0.3), \mathbf{F}-$ MACE in comparison between patients with or without bifurcation at target lesion $(p=0.6)$ 
A

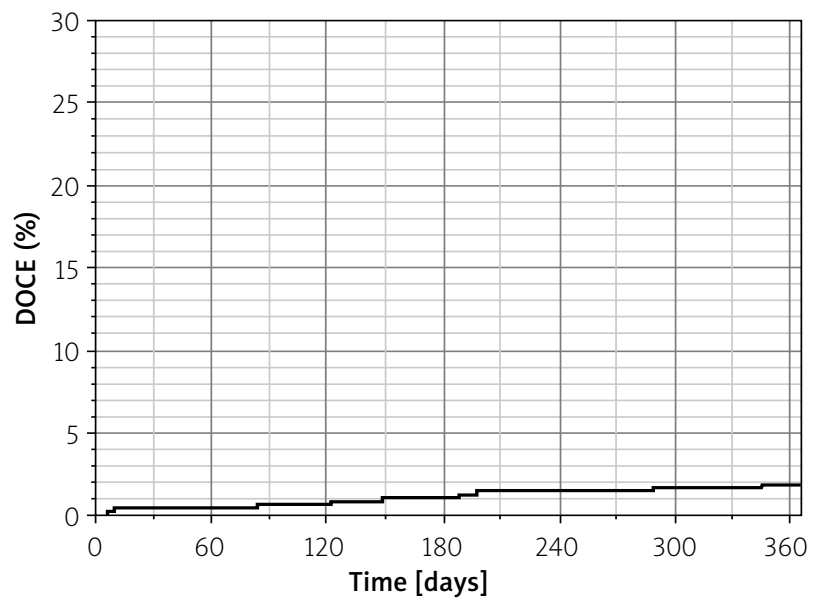

C

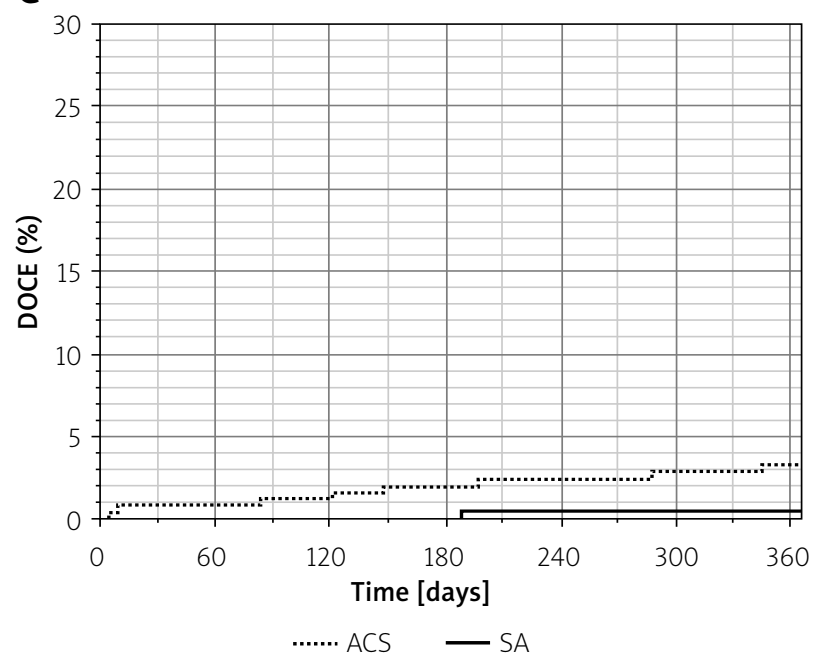

E

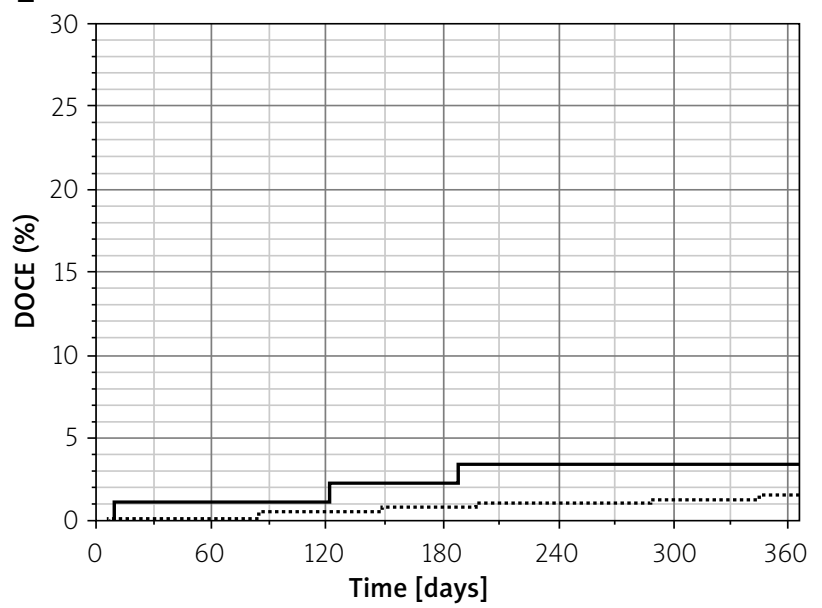

....... No calcification and mild - Moderate and heavy
B

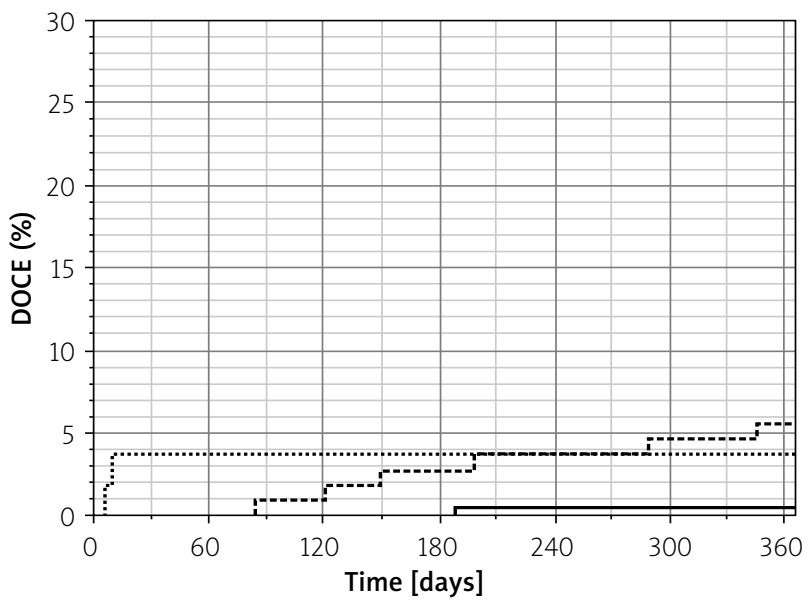

....... STEMI - - NSTEMI

_ Stable angina [.... Unstable angina

D

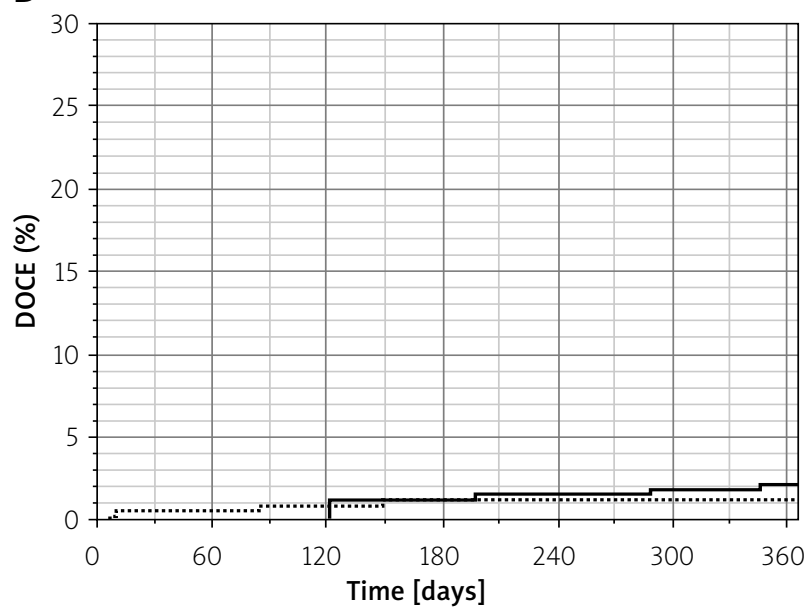

....... Not tortuous and mild _ Moderate and severe

$\mathrm{F}$

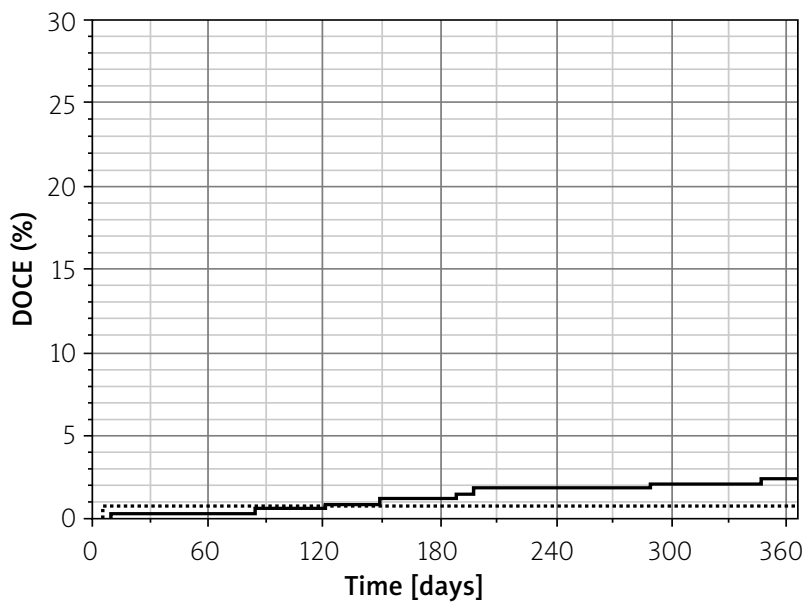

….... Yes - No

Figure 2. Kaplan-Meier curves for device-oriented composite endpoint incidence: $A$ - DOCE in all included patients, B - DOCE in comparison of PCl indications $(p<0.006), \mathbf{C}-$ DOCE in comparison of ACS vs. SA $(p<0.03)$, D - DOCE in comparison between patients with no or mild versus moderate or severe tortuosity at target vessel $(p=0.6), E-D O C E$ in comparison patients with no or mild versus moderate or heavy calcification at target vessel $(p=0.3), \mathbf{F}-$ DOCE in comparison between patients with or without bifurcation at target lesion $(p=0.3)$ 
Table II. Clinical outcome, device-oriented composite endpoint and major adverse cardiovascular events after 12 months of observation in comparison between patients with no or mild and moderate or severe tortuosity of target vessel. Follow-up was available in 468 patients. Total MACE number is not equal to added components. Several events may occur in 1 patient

\begin{tabular}{lccc} 
Parameter & No and mild tortuosity $(n=387)$ & Moderate and severe tortuosity $(n=78)$ & $P$-value \\
\hline DOCE & $8(2.08 \%)$ & $1(1.28 \%)$ & 0.6 \\
\hline MACE & $11(2.86 \%)$ & $3(3.85 \%)$ & 0.6 \\
\hline Death & $1(0.26 \%)$ & $1(1.28 \%)$ & 0.3 \\
\hline MI & $6(1.55 \%)$ & $2(2.56 \%)$ & 0.6 \\
\hline TLR with urgent PCl & $7(1.81 \%)$ & $0(0 \%)$ & 0.6 \\
\hline TVR with urgent CABG & $0(0 \%)$ & $0(0 \%)$ & - \\
\hline Stent thrombosis & $2(0.52 \%)$ & $0(0.00 \%)$ & 1.0 \\
\hline Stent restenosis & $9(2.33 \%)$ & $1(1.28 \%)$ & 1.0
\end{tabular}

$C A B G$ - coronary artery bypass grafting, DOCE - device-oriented composite endpoint, MACE - major adverse cardiovascular event, MI - myocardial infarction, $P C l$ - percutaneous coronary interventions, $T L R$-target lesion revascularisation, TVR-target vessel revascularisation.

Table III. Clinical outcome, device oriented composite endpoint and major adverse cardiovascular events after 12 months observation in comparison between patients with no or mild and moderate or severe calcification of target vessel. Follow-up was available in 468 patients. Total MACE number is not equal to added components. Few events may occur in 1 patients

\begin{tabular}{lccc} 
Parameter & No and mild calcification $(n=380)$ & Moderate and severe calcification $(n=86)$ & $P$-value \\
\hline DOCE & $6(1.59 \%)$ & $3(3.49 \%)$ & 0.3 \\
\hline MACE & $10(2.64 \%)$ & $4(4.65 \%)$ & 0.3 \\
\hline Death & $1(0.26 \%)$ & $1(1.16 \%)$ & 0.3 \\
\hline MI & $5(1.32 \%)$ & $3(3.49 \%)$ & 0.2 \\
\hline TLR with urgent PCl & $5(1.32 \%)$ & $2(2.33 \%)$ & 0.6 \\
\hline TVR with urgent CABG & $0(0 \%)$ & $0(0 \%)$ & - \\
\hline Stent thrombosis & $1(0.26 \%)$ & $1(1.16 \%)$ & 0.3 \\
\hline Stent restenosis & $8(2.11 \%)$ & $2(2.33 \%)$ & 1.0
\end{tabular}

$C A B G$ - coronary artery bypass grafting, DOCE - device-oriented composite endpoint, MACE - major adverse cardiovascular event, MI - myocardial infarction, $P C l$ - percutaneous coronary interventions, TLR - target lesion revascularisation, TVR-target vessel revascularisation.

$31.9 \%$ [17]. The above-mentioned higher rate of events in the UA group could be coincidental or the result of significantly lower prasugrel usage in comparison with other patients included in our study. The current use of BVS is still restricted to stable patients and non-complex lesions. A recent study showed good results in an unselected population including lesions with higher complexity, but the small sample size and short observation period are the main limitations of that study [23]. Other large studies including real-world patients with more complex lesions demonstrated optimal clinical outcome and efficacy comparable with that found in our research $[12,14,24]$. Proper lesion preparation and assessment by intra-coronary imaging may play an important role in treatment of ACS and lesions of higher complexity.
The major limitations of this prospective study are the non-randomised manner and all the known drawbacks of single-arm registry studies. Early outcomes in the presented registry are promising but do not exclude a significant increase of incidence of events in the longer term. This study was insufficient to examine low-frequency events such as cardiac death and stent thrombosis. Longer follow-up is required to assess occurrence of complications after BVS implantation. There is a potential bias caused by the loss of patients during follow-up and lack of some data.

\section{Conclusions}

Bioresorbable vascular scaffold can be successfully and safely used for ACS treatment and in lesions of high- 
Table IV. Clinical outcome, device-oriented composite endpoint and major adverse cardiovascular events after 12 months of observation in comparison between patients with and without bifurcation of target vessel. Follow-up was available in 468 patients. Total MACE number is not equal to added components. Several events may occur in 1 patients

\begin{tabular}{lccc} 
Parameter & $\begin{array}{c}\text { Bifurcation } \\
(n=127)\end{array}$ & $\begin{array}{c}\text { No bifurcation } \\
(n=339)\end{array}$ & $P$-value \\
\hline DOCE & $1(0.79 \%)$ & $8(2.36 \%)$ & 0.3 \\
\hline MACE & $3(2.36 \%)$ & $11(3.26 \%)$ & 0.6 \\
\hline Death & $1(0.79 \%)$ & $1(0.29 \%)$ & 0.5 \\
\hline MI & $2(1.57 \%)$ & $6(1.78 \%)$ & 0.9 \\
\hline TLR with urgent PCI & $0(0.00 \%)$ & $7(2.07 \%)$ & 0.1 \\
\hline TVR with urgent CABG & $0(0 \%)$ & $0(0 \%)$ & - \\
\hline Stent thrombosis & $0(0.00 \%)$ & $2(0.59 \%)$ & 0.4 \\
\hline Stent restenosis & $0(0.00 \%)$ & $10(2.96 \%)$ & 0.051
\end{tabular}

CABG - coronary artery bypass grafting, DOCE - device-oriented composite endpoint, MACE - major adverse cardiovascular event, $M I-$ myocardial infarction, $P C l$ - percutaneous coronary interventions, $T L R$ - target lesion revascularisation, TVR - target vessel revascularisation.

er complexity such as calcified lesions, severe tortuous vessels or bifurcation in the target vessel. The presented data are promising, but further investigation and longerterm clinical follow-up of patients are required.

\section{Conflict of interests}

The authors declare no conflict of interest.

\section{References}

1. Karabulut A, Demirci Y. Cutting balloon use may ease the optimal apposition of bioresorbable vascular scaffold in in-stent stenosis. Postep Kardiol Inter 2015; 11: 64-6.

2. Rama-Merchan JC, Mattesini A, Dall'Ara G, et al. Chronic total occlusion successfully treated with a bioresorbable everolimus-eluting vascular scaffold. Postep Kardiol Inter 2014; 10: 128-39.

3. Onuma Y, Serruys PW, Perkins LEL, et al. Intracoronary optical coherence tomography and histology at 1 month and 2, 3, and 4 years after implantation of everolimus-eluting bioresorbable vascular scaffolds in a porcine coronary arterymodel: an attempt to decipher the human optical coherence tomography images in the ABSORB trial. Circulation 2010; 122: 2288-300.

4. Bruining N, de Winter S, Roelandt JRTC, et al. Monitoring in vivo absorption of a drug-eluting bioabsorbable stent with intravascular ultrasound-derived parameters a feasibility study. JACC Cardiovasc Interv 2010; 3: 449-56.

5. Lesiak M, Araszkiewicz A. Leaving nothing behind: is the bioresorbable vascular scaffold a new hope for patients with coronary artery disease? Postep Kardiol Inter 2014; 10: 283-98.

6. Dariusz Dudek D, Rzeszutko $t$, Zasada W, et al. Bioresorbable vascular scaffolds in patients with acute coronary syndromes: the POLAR ACS study. Pol Arch Med Wewn 2014; 124: 669-77.
7. Cortese B, Ielasi A, Varricchio A, et al. Registro Absorb Italiano (BVS-RAI): an investigators-owned and -directed, open, prospective registry of consecutive patients treated with the Absorb ${ }^{\mathrm{TM}}$ BVS: study design. Cardiovasc Revasc Med 2015; 16: 340-3.

8. Gori T, Schulz E, Hink U, et al. Clinical, angiographic, functional, and imaging outcomes 12 months after implantation of drug-eluting bioresorbable vascular scaffolds in acute coronary syndromes. JACC Cardiovasc Interv 2015; 8: 770-7.

9. Rzeszutko $t$, Siudak Z, Włodarczak A, et al. Use of bioresorbable vascular scaffolds in patients with stable angina and acute coronary syndromes. Polish National Registry. Kardiol Pol 2014; 72: 1394-409.

10. Cutlip DE, Windecker S, Mehran R, et al. Clinical end points in coronary stent trials: a case for standardized definitions. Circulation 2007; 115: 2344-351.

11. Giuseppe G, Ortega-Paz L, Brugaletta S, et al. Bioresorbable vascular scaffold implantation in acute coronary syndromes: clinical evidence, tips and tricks. Postep Kardiol Inter 2015; 11: 161-9.

12. Ishibashi Y, Nakatani S, Sotomi Y et al. Relation between bioresorbable scaffold sizing using QCA-Dmax and clinical outcomes at 1 year in 1,232 patients from 3 study cohorts (ABSORB Cohort $B, A B S O R B$ EXTEND, and ABSORB II). JACC Cardiovasc Interv 2015; 8: 1715-26.

13. Wöhrle J, Naber C, Schmitz T, et al. Beyond the early stages: insights from the ASSURE registry on bioresorbable vascular scaffolds. Eurolntervention 2015; 11: 149-56.

14. Capodanno D, Gori T, Nef H, et al. Percutaneous coronary intervention with everolimus-eluting bioresorbable vascular scaffolds in routine clinical practice: early and midterm outcomes from the European multicentre GHOST-EU registry. Eurolntervention 2015; 10: 1144-53.

15. Cassese S, Byrne RA, Ndrepepa G, et al. Everolimus-eluting bioresorbable vascular scaffolds versus everolimus-eluting metallic stents: a meta-analysis of randomised controlled trials. Lancet 2016; 387: 537-44.

16. Kimura T, Kozuma K, Tanabe K, et al. A randomized trial evaluating everolimus-eluting Absorb bioresorbable scaffolds vs. everolimus-eluting metallic stents in patients with coronary artery disease: ABSORB Japan. Eur Heart J 2015; 36: 3332-42.

17. Sabaté $M$, Windecker $S$, Iñiguez $A$, et al. Everolimus-eluting bioresorbable stent vs. durable polymer everolimus-eluting metallic stent in patients with ST-segment elevation myocardial infarction: results of the randomized ABSORB ST-segment elevation myocardial infarction-TROFI II trial. Eur Heart J 2016; 37 : 229-40.

18. Brugaletta S, Gori T, Low AF, et al. Absorb bioresorbable vascular scaffold versus everolimus-eluting metallic stent in ST-segment elevation myocardial infarction: 1-year results of a propensity score matching comparison: the BVS-EXAMINATION Study (Bioresorbable Vascular Scaffold-A Clinical Evaluation of Everolimus Eluting Coronary Stents in the Treatment of Patients With ST-segment Elevation Myocardial Infarction). JACC CardiovasC Interv 2015; 8: 189-97.

19. Abizaid A, Costa Jr. JR, Bartorelli AL, et al. The ABSORB EXTEND study: preliminary report of the twelve-month clinical outcomes in the first 512 patients enrolled. Euro Intervention 2015; 10: 1396-401.

20. Costopoulos C, Latib A, Naganuma T, et al. Comparison of early clinical outcomes between Absorb bioresorbable vascular scaffold and everolimus-eluting stent implantation in a real-world population. Catheter Cardiovasc Interv 2015; 1: E10-5. 
21. Mattesini A, Secco GG, Dall'Ara G, et al. ABSORB biodegradable stents versus second-generation metal stents: a comparison study of 100 complex lesions treated under OCT guidance. JACC Cardiovasc Interv 2014; 7: 741-50.

22. Caiazzo G, Kilic ID, Fabris E, et al. Absorb bioresorbable vascular scaffold: what have we learned after 5 years of clinical experience? Int J Cardiol 2015; 15: 129-36.

23. Jaguszewski M, Ghadri JR, Zipponi M, et al. Feasibility of second-generation bioresorbable vascular scaffold implantation in complex anatomical and clinical scenarios. Clin Res Cardiol 2015; 2: 124-35.

24. Robaei D, Back LM, Ooi SY, et al. Everolimus-eluting bioresorbable vascular scaffold implantation in real world and complex coronary disease: procedural and 30-day outcomes at two Australian centres. Heart Lung Circ 2015; 9: 854-9. 